\title{
Research on Teaching Reform of College Practice Course Based on "4C" Model
}

\section{Shaorong Wei, Xiaoying Wang, Zhiqiang Liu and Lu Wang}

Qinghai University, Department of Computer Technology and Application, Xining 810016, China

\begin{abstract}
University practice teaching is an important Experiment practice step in college quality education system. It can cultivate the high quality of various types of engineering and technical personnel with innovative ability, to meet the needs of economic and social development. But at present, there are many problems in the course of college practice, such as the single teaching mode, the cognitive process of the students, and so on. The paper puts forward the innovation of the teaching reform of college practice course based on the "4C" model. The practical results show that the application of " $4 \mathrm{C}$ " teaching mode in the practice course teaching and training students' ability to solve the problem of independent design, and experience a sense of achievement, inspire students to create, explore, discover and constantly learning. It can improve students' practice and innovation ability.
\end{abstract}

Keywords: 4C; Teaching model; Practice course

\section{大学实验课程教学中 “ $4 \mathrm{C”}$ 模式的应用研究}

\author{
魏绍蓉，王晓英，刘志强，王璐 \\ （青海大学 计算机技术与应用系，青海 西宁 810016）
}

摘要: 大学实验课程教学对于培养创新能力强、适应经济社会发展需要的高质量各类型工程技术人才是非常重要的教学 环节。但是目前大学实验课程存在教学方式单一、脱离学生的认知过程等诸多问题。文章创新性地提出 “ $4 \mathrm{C}$ ” 模式在大学 实验课程教学中的应用。实践结果表明, “4C”模式在实验课程教学中的应用, 培养学生独立设计问题的解决方案的能力, 体验成就感, 激励学生去创造、探索、发现并不断学习, 提高学生的实践及创新能力培养。

关键词: $4 \mathrm{C}$; 教学模式; 实验课程

中图分类号: G642.2 文献标识码: A

\section{引言}

实验是科学的基础, 是科学发展的动力源泉, 是解决学生学习兴趣低, 知识面窄, 发现问题、分析问 题与解决问题以及动手操作能力的关键, 所以实验教学在现代教学中占有重要的地位。大学实验教学过程 应以学生的全面发展为本, 突出在实验教学中的主体地位, 通过实验教学使学生更直观地了解所学理论知 识, 引发学生的创新能力。推动学生探究性学习和合作性学习, 树立了学生的合作精神和科学的价值观教 育。在实验教学环节中, 如果学习者所处的学习环境能恰到好处地给学习者提供挑战, 就能激励学习者去 探索、理解、不断学习, 并体验到一种高度享受感。科学研究表明, 这种状态正是学习效果最佳的状态, 而实践证明 “ $4 \mathrm{C}$ ”模式在实验教学中的应用可以使学习者达到学习效果最佳状态 ${ }^{[1]}$ 。

\section{1 “ $4 \mathrm{C}$ ”模式}

“4C” 模式起源于建构主义。“建构主义” 是瑞士认知发展领域最有影响的心理学家皮亚杰提出：知 识是建构的, 而不是传播的。让学生自己动手实践, 建构知识; 指导教学是以教师为中心, 实践教学以学 生为中心; 让学生用不同的方式展示所学 [2]。

“4C” 模式, 联系 (connect)、建构 (construct)、反思 (contemplate) 和延续 (continue)。

联系 (connect) : 如果学生能够把新的体验与已有的 “经验库” 联系起来, 从而激发起他们了解更多 
知识的愿望, 那么就能取得更好的学习效果。

建构 (construct) : 通过在真实世界中建构事物, 进而在头脑中组合知识。让学生思考、策划, 最终 形成个人解决方案。

反思 (contemplate) : 促使学生花时间思考所看到或所建构的实物, 从而对自己的体验加深理解。让 学生对方案展开讨论、反思并调整自己的想法, 教师则以提问的方式推进这一过程。

延续 (continue) : 延续建立在学生渴求了解更多的欲望之上。寻求学习更多技能的愿望会将其带到一 个新的 “联系” 阶段, 使他们在一个螺旋式上升的良性循环过程中, 超越难度不断增加的挑战。

“4C” 模式能使学生参与到真实世界的设计挑战中，思考并找出创造性解决问题的方法。团队协作， 分享思想和知识, 参与动手操作完成实验任务, 面对新挑战充满信心, 有乐趣并快乐地学习。

\section{2 “ $4 \mathrm{C}$ ”教学在大学实验课程教学中的应用实施方案}

利用 “4C” 模式进行实验课程教学, 使实践环节更具交互性、协作性, 实验室就是一个研讨室, 学生 在教师指导下聚在一起交换想法和观点。教师要注重学生在实践中的主体地位, 指导学生在深入掌握课程 理论知识的基础上, 形成良好的实践操作能力, 促进学生的全面发展, 培养工程实践能力为核心的工程应 用型人才。

\section{1 设计任务, 构思、设计实验任务, 促使学生把实践体验与已有的知识联系起来}

教师根据教学内容构思、创新、设计实验任务, 促使学生把新的体验与已有的知识联系起来, 激发学 生的思维兴趣和创作兴趣及对新知识、新方法的深度探求。在实验任务的设计过程中, 教师首先要根据分 析和挖掘教学中的主要知识点, 设计一些具有趣味性、竞技、目的性的任务, 并将这些专业知识内容贯穿 于任务的始终, 带领更多的学生进入到课程的情景之中。在充分调动学生参与的积极性和主动性的同时, 使学生不断加深对各类知识内容的深入掌握和灵活运用, 并形成一定的空间结构框架和思维能力。教师通 过设计任务, 启发学生将知识点与任务有机结合, 充分检验学生的学习水平和应用能力, 使得学习更具交 互性、协作性。在此教学过程中, 教师只是一名经验丰富的教学促进者, 学生在其指导下聚在一起交换想 法和观点, 加强创新性思维和扩展能力的锻炼 [3]。

\section{2 分组实施, 合理分组, 让学生在实施与探索中假设、验证, 完成知识的自我建构}

教师在实验课程中遵循同组异质、异组同质的原则 [4-5] 对学生进行分组, 让学生在实践的同时分析、 思考、合作, 重新构建知识, 这样才能主动准确地学习知识。同组异质, 包括性别差异、思维差异、动手 能力差异、性格差异等, 力求形成互补型合作团队, 便于发挥小组各成员的个性特长。异组同质, 则是保 持组际之间的均衡性, 有利于组际间的交流和竞争, 有利于对各组实践活动的评价。将不同差异的学生搭 配分组, 能言善辩的学生和沉默葟言的学生搭配, 善于思考分析的学生和喜欢动手操作的学生搭配。通常 是 3-5 人为一组, 人数不易多, 这样有利于积极的相互支持与配合, 有利于面对面促进性互动。要保证各 组实力接近, 这样各组之间才具有可比性, 有利于学生之间互相帮助、互相促进、共同提高, 同时活动进 度相对一致。

每一个实验任务都有建构活动。在建构阶段，学生通过分析、思考、运用知识完成既定任务，给学生 提供”涉身认知”的机会。通过任务活动, 大脑的高级思维潜能被激发, 学生可以深入理解事物的因果关系, 掌握事物运作的规律, 从而达到课程要求的“知识建构”目标。。 


\section{3 引导课堂, 师生共同引导课堂发展, 完成学生的反思阶段}

反思阶段促使学生花时间思考他们所看到或所建构的实物, 从而对自己的体验加深理解。83\%的专家 预测, 教育将变得更具个性化, 教师的传统角色也被期望产生变化, 教师完全主导的角色正在向师生共同 引导课堂发展。在学习过程中, 教师要观察学生参与合作学习的情况, 做好巡回指导。对操作中发现的错 误进行引导并给学生和小组提供帮助 [6-7], 调动全员参与讨论, 让每一位学生都参与其中。教师还要积 极协调人际关系, 减少和避免组内、组间冲突, 特别要对合作不协调的小组或合作有困难的小组给予及时 的帮助和引导, 并积极指导学生进行自我监控, 使每个小组都能在学习中有所收获, 按时完成合作学习任 务。

对学生的巡查和调控不能过于频繁, 以免学生丧失信心, 过分依赖教师。学生在生理发展和心理特征 上的差异是客观存在的, 要针对学生的个体差异, 进行灵活的、符合学生实际的差异性辅导, 教师应给予 学生适当的激励, 注重指导学生的实践操作能力, 让学生对实践方案展开讨论、反思并调整自己的想法, 教师则以提问的方式推进这一过程。学生在实践中体会到自我成就感, 当学生体会成功的喜悦, 就会对学 习充满兴趣和信心。

\section{4 评价, 多种评价机制结合促使学生有寻求更多知识的愿望, 形成新的 “联系”, 延续}

“ $4 \mathrm{C}$ ” 模式在实验课程中的创新应用, 评价机制地改进至关重要。学校教育不应是 “批量化生产” 只 会读书、只会做题、只会考试的机器, 而更应该是着眼于培养能明辩事理、有大爱胸襟、敢于担当、心智 健全, 既全面发展又学有所长, 并终身可持续发展的合格的社会公民。在 “ $4 \mathrm{C}$ ” 模式中改变传统的单向评 价机制, 不仅是教师对学生的评价, 也要有学生与学生之间的评价, 这种评价活动能够有效地检测每一位 学生的知识获取水平和实践操作能力, 让学生以一个欣赏者的姿态, 真正进入到亲身经历、主动思考、实 践、创造的角色中, 实现自我价值, 体验成就感。

评价的方式可以是过程性评价、反思评价和发展性评价 [8-11]。过程评价主要是对学生在任务实施的 过程中, 每一个阶段的实践操作能力, 团队协作意识等内容进行评价。反思性评价主要是在学生完成任务 后, 对任务实施的过程中，对技术上的自我评价和主要感想进行述说。发展性评价是展现学生个人潜力和 创新性思维的重要平台, 学生阐述在整个任务实施过程中产生的创新性想法, 教师对学生的想法进行相对 引导。

展示实验任务结果, 学生全体参与, 让每个参与者都有成就感。在实践过程中, 小组作为一个整体, 在学习反馈交流时, 小组成员全员参与, 各抒已见, 使之有一定的责任, 产生动力。同时鼓励和引导小组 间开展竞争，良好健康的竞争使学生产生积极向上的动力。小组竞争会促进小组成员之间的分工合作增加 默契, 并逐渐形成各小组的特色。在竞争中, 教师要关注团队成员的参与、分工、合作、协调, 并及时鼓 励。

\section{3 结束语}

基于 “ $4 \mathrm{C}$ ”模式的大学实践教学改革, 让学习者在互动中感觉到试错, 在试错中学习; 传统教学中以 教师为中心转变为以学生为中心; 实践内容以课本为中心转变为以 “任务” 为中心, 以课堂为中心转变为 以实际经验为中心。 “ $4 \mathrm{C}$ ” 模式把实验室交给学生, 深入挖掘和提升学生的实践操作能力, 并且完成情感、 知识、智慧、价值观的碰撞与沟通。基于 “ $4 \mathrm{C}$ ” 模式的大学实践教学改革, 带给学生巨大而持久的学习动 
力, 促使学生掌握变革、创新、团队合作等未来竞争的核心要素, 成为具有工程实践能力的综合型应用人 才。

\section{4 致谢}

基金项目：国家自然科学基金项目（No.61363019）；青海省计算机基础课程教研创新团队 项目（No. 201509013 ); 青海省骨干教师培养计划; 青海大学教育教学改革与建设课题 (2014JY01)

Fund Project: National Natural Science Foundation of China (No.61363019); Qinghai Province Computer Basic Course Teaching and Research Innovation Team Project (No.201509013); Qinghai Province Key Teacher Training Program; Qinghai University Education and Teaching Reform and Construction Project (2014JY01)

\section{参考文献:}

[1] 李新房, 李静, 邱婧玲. 4C/ID 模式在教育技术学专业课程教学中的应用研究一一《PhotoshopCS4》课程为例 [J]. 福 建电脑, 2015, 01:46-47.

[2] 曲延华, 程立英, 张玉梅. 基于 4C/ID 模式的《模拟电子技术》课程教学设计研究 [J]. 沈阳师范大学学报 (自然科学 版), 2014, 02: 296-299.

[3] 厉旭云, 梅汝焕, 叶治国, 富丽琴, 夏强, 陆源. 高校实验教学研究的发展及趋势 [J]. 实验室研究与探 索, 2014, 33 (03) : 131-135+197.

[4] 向佐军. 四要素教学设计 (4C/ID) 模式述评 [J]. 现代基础教育研究, 2015, 02:202-207.

[5] 何朝阳, 欧玉芳, 曹祁. 美国大学翻转课堂教学模式的启示 [J]. 高等工程教育研究, 2014, 02:148-151+161.

[6] 何文广, 周珂, 熊刚强. 程序设计课程实验教学改革与实践 [J]. 实验室研究与探索, 2016, 35 (06) : 163-169.

[7] 林青松. 基于翻转课堂的 “现代教育技术” 实验课程设计 [J]. 实验室研究与探索, 2016, 33 (01) : 194-198.

[8］程万里. 用乐高 4C 教学法 “建构” 信息技术课堂 [J]. 学子 (理论版), 2015, 09:9.

[9] 孙雨生,程亚南, 朱礼军. 基于 MOOC 的高校教学模式构建研究 [J]. 远程教育杂志, 2015, 03:65-71.

[10]李红美, 陆国栋, 张剑平. 后 MOOC 时期高等学校教学新模式探索 [J]. 高等工程教育研究, 2014, 06 :58-67.

[11] 王建宏, 常俊英, 梁存珍, 周翠红. 以学生为中心的实验室安全和文化建设 [J]. 实验室研究与探 索, 2016, 35 (06) :288-292.

\section{References:}

[1] Li Xinfang, Li Jing, Qiu Jingling. Application of 4C / ID Model in the Teaching of Educational Technology Specialty Course - Taking "PhotoshopCS4" Course as an Example [J]. Fujian Computer, 2015, 01: 46- 47.

[2] Qu Yanhua, Cheng Liying, Zhang Yumei. Study on Teaching Design of "Analog Electronic Technology" Course Based on 4C / ID Mode [J]. Journal of Shenyang Normal University (Natural Science Edition), 2014, 02: 296-299.

[3] Li Xuyun, Mei Ruhuan, Ye Zhiguo, Fu Liqin, Xia Qiang, Lu Yuan. Study on the development and trend of experimental teaching research in colleges and universities [J]. Laboratory Research and Exploration, 2014, 33 (03): 131-135 + 197.

[4] Zuo Jun. Four elements of the teaching design (4C / ID) model review [J]. Modern basic 
education research, 2015, 02: 202-207.

[5] He Chaoyang, Ouyu Fang, Cao Qi. American university to flip classroom teaching mode of enlightenment [J]. Higher Engineering Education Research, 2014, 02: 148-151 + 161.

[6] He Wenguang, Zhou Ke, Xiong Gangqiang. Program design curriculum experiment teaching reform and practice [J]. Laboratory research and exploration, 2016, 35 (06): 163-169.

[7] Lin Qingsong. Based on the flip classroom "modern educational technology" experimental course design [J]. Laboratory research and exploration, 2016, 33 (01): 194-198.

[8] Cheng Wanli. Using LEGO 4C teaching method to "construct" information technology classroom [J]. Students (theoretical), 2015, 09: 9.

[9] Sun Yusheng, Cheng Yannan, Zhu Lijun. MOOC-based teaching model construction research [J]. Distance Education Journal, 2015, 03: 65-71.

[10] Li Hongmei, Lu Guodong, Zhang Jianping. After the MOOC period of higher education teaching model [J]. Higher Engineering Education Research, 2014, 06: 58-67.

[11] Wang Jianhong, Chang Junying, Liang Cunzhen, Zhou Cuihong. Student-centered laboratory safety and cultural construction [J]. Laboratory Research and Exploration, 2016, 35 (06): 288-292. 